\title{
The Early-phase Distribution of the Milky Way Using K-giant Stars From LAMOST DR5
}

\author{
Ali Taani * \\ Physics Department, Faculty of Science, Al-Balqa Applied University,Jordan
}

\begin{abstract}
With the advent of large astronomical surveys, the need of identification of the most interesting astrophysical object is required. In this work we focus on extracting red giant metal-poor stars from the LAMOST DR5. We then query the 5-D space coordinates from the Gaia DR2 to investigate their energy-action space. This sample will be used for studying the chemical and dynamical evolution of the early galactic phase. Also, identifying stars with kinematical similarities would enable us to trace the galactic assembly.
\end{abstract}

\section{Keywords: Galaxy: halo-stars: kinematics and dynamics-galaxies: structure}

\section{1. large data surveys}

The next wave of astronomical surveys will substantially increase the number of observed stars in the Milky Way (Mardini et al., 2019b, Mardini. et al., 2019c, Masda et al., 2019, Taani et al., 2019a,b,c, 2020). Part of the challenge is how to efficiently select the most exciting targets for spectroscopic to follow-up, especially when dealing with photometric surveys, where all the information on physical parameters and chemistry of stars is encoded in their observed colors. The immediate future involve (i) identification of low-metallicity star candidates from photometry and low/medium resolution spectroscopy, (ii) high-resolution spectroscopic follow-up to determine detailed chemical abundance patterns, (iii) compare observations with results from theoretical and computational models, and v) computationally tracing the kinematic history and orbital evolution of these stars with Gaia data (Abdusalam et al., 2020, Mardini et al., 2019a, Mardini et al., 2020, Placco et al., 2020).

Large data surveys usually provide low-resolution $(R \simeq 400)$ spectra that can be used to examine the strength of the CaII $\mathrm{K}$ line and thus obtain a first estimate of the metal content of the star. However, some surveys provided mixture resolution (low and medium-resolution). The Large Sky Area Multi-Object Fiber Spectroscopic Telescope (LAMOST) survey is a example of such surveys. In its all phases (pilot survey + six years surveys) provided spectra for 8,966,416 stars.

The HK survey and the Hamburg/ESO (HES) survey are other surveys of this low-resolution surveys, that were prolific sources of metal-poor stars. The HK-II survey aimed at identifying the metal-poor red giant cool stars that may have been missed in the original analysis due to the temperature bias against cool stars. It allowed to raise the efficiency of the HK survey to detect metal-poor stars from 11 to $32 \%$.

Application of mathematical clustering algorithms to the space of orbital energies and other suitable dynamical parameters will result in stars being grouped based on the similarity of their orbits around the Galaxy. Identification of dynamically tagged groups in large catalogs of very metal-poor will provide another means to identify their likely progenitor environments.

We applied some sophisticated cuts on the fifth data release of the Large Sky Area Multi-Object Fibre Spectroscopic Telescope (LAMOST DR5) to efficiently select, astrophysically important, Kgiants stars. We queried in about 2000 stars. Further, we crossed-match the LAMOST K-giant sample

*ali.taani@bau.edu.jo 


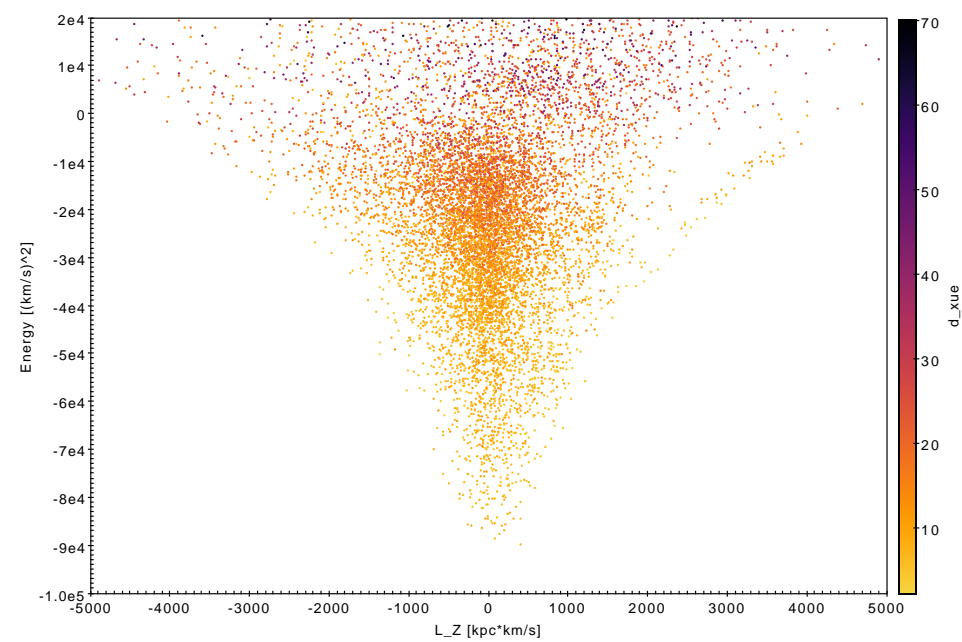

Figure 1. The distribution of K-giant stars (2000) adopted from LAMOST DR5 in the energy-action space. Color-coded by their spectroscopic metallicities $[\mathrm{Fe} / \mathrm{H}]$.

with the second Gaia data release to query full 6 dimensional phase-space coordinates. Actions and total orbital energy were calculated with galpy using the 'MW- Potential2014' potential (see Figure $1)$.

\section{Future Recommendation/work}

In future work we will identify potential dynamical groups in our sample. These groups are crucial for the study of the galactic assembly. We expect to determine the role of K-giant stars as tracers of the dynamical evolution of the Milky Way.

\section{Acknowledgements}

This work has made use of data from the European Space Agency (ESA) mission Gaia (https://www.cosmos.esa.int/ gaia), processed by the Gaia Data Processing and Analysis Consortium (DPAC, https://www.cosmos.esa.int/web/gaia/dpac/ consortium). Funding for the DPAC has been provided by national institutions, in particular the institutions participating in the Gaia Multilateral Agreement.

\section{References}

Abdusalam K., Ablimit I., Hashim P., Lü G. L., Mardini M. K., Wang Z. J., 2020, Astrophys. J., 902, 125

Mardini M. K., et al., 2019a, The Astrophysical Journal, 875, 89

Mardini M. K., Placco V. M., Taani A., Li H., Zhao G., 2019b, The Astrophysical Journal, 882, 27

Mardini. M. K., Ershiadat N., Al-Wardat M. A., Taani A. A., Özdemi S., Al-Naimiy H., Khasawneh A., 2019c, in Journal of Physics Conference Series. p. 012024 (arXiv:1904.09608), doi:10.1088/1742-6596/1258/1/012024

Mardini M. K., et al., 2020, arXiv e-prints, p. arXiv:2009.12142

Masda S. G., Docobo J. A., Hussein A. M., Mardini M. K., Al-Ameryeen H. A., Campo P. P., Khan A. R., Pathan J. M., 2019, Astrophysical Bulletin, 74, 464

Placco V. M., Santucci R. M., Yuan Z., Mardini M. K., Holmbeck E. M., Wang 2020, Astrophys. J., 897, 78

Taani A., Karino S., Song L., Al-Wardat M., Khasawneh A., Mardini M. K., 2019a, Research in Astronomy and Astrophysics, 19, 012

Taani A., Abushattal A., Mardini M. K., 2019b, Astronomische Nachrichten, 340, 847

Taani A., Karino S., Song L., Mardini M., Al-Wardat M., Abushattal A., Khasawneh A., Al-Naimiy H., 2019c, in Journal of Physics Conference Series. p. 012029, doi:10.1088/1742-6596/1258/1/012029

Taani A., Khasawneh A., Mardini M., Abushattal A., Al-Wardat M., 2020, arXiv e-prints, p. arXiv:2002.03011 